\title{
Quantifying consistent individual differences in habitat selection
}

\author{
Martin Leclerc $^{1} \cdot$ Eric Vander Wal $^{1,2} \cdot$ Andreas Zedrosser $^{3,4} \cdot$ Jon E. Swenson $^{5,6}$. \\ Jonas Kindberg $^{7} \cdot$ Fanie Pelletier $^{1}$
}

Received: 20 April 2015 / Accepted: 27 October 2015 / Published online: 23 November 2015

(C) The Author(s) 2015. This article is published with open access at Springerlink.com

\begin{abstract}
Habitat selection is a fundamental behaviour that links individuals to the resources required for survival and reproduction. Although natural selection acts on an individual's phenotype, research on habitat selection often pools inter-individual patterns to provide inferences on the population scale. Here, we expanded a traditional approach of quantifying habitat selection at the individual level to explore the potential for consistent individual differences of habitat selection. We used random coefficients in resource selection functions (RSFs) and repeatability estimates to test for variability in habitat selection. We applied our method to a detailed dataset of GPS relocations of brown bears (Ursus arctos) taken over a period of 6 years, and assessed whether they displayed repeatable individual differences in habitat selection toward two habitat types: bogs and recent timber-harvest cut blocks. In our analyses, we controlled for the availability of habitat,
\end{abstract}

Communicated by Ilpo Kojola.

Electronic supplementary material The online version of this article (doi:10.1007/s00442-015-3500-6) contains supplementary material, which is available to authorized users.

Andreas Zedrosser

andreas.zedrosser@hit.no

Martin Leclerc

Martin.Leclerc2@USherbrooke.ca

1 Département de Biologie, Canada Research Chair in Evolutionary Demography and Conservation and Centre for Northern Studies, Université de Sherbrooke, Sherbrooke J1K2R1, Canada

2 Department of Biology, Memorial University of Newfoundland, St. John's, NL A1B 3X9, Canada

3 Department of Environmental and Health Studies, Faculty of Arts and Sciences, Telemark University College, $3800 \mathrm{~B} \emptyset \mathrm{i}$ Telemark, Norway i.e. the functional response in habitat selection. Repeatability estimates of habitat selection toward bogs and cut blocks were 0.304 and 0.420 , respectively. Therefore, 30.4 and $42.0 \%$ of the population-scale habitat selection variability for bogs and cut blocks, respectively, was due to differences among individuals, suggesting that consistent individual variation in habitat selection exists in brown bears. Using simulations, we posit that repeatability values of habitat selection are not related to the value and significance of $\beta$ estimates in RSFs. Although individual differences in habitat selection could be the results of non-exclusive factors, our results illustrate the evolutionary potential of habitat selection.

Keywords Functional response - Personality · Repeatability $\cdot$ Ursus arctos

\section{Introduction}

Understanding factors that shape animals' habitat selection is a fundamental ecological challenge (Morris 2011),

4 Department of Integrative Biology, Institute of Wildlife Biology and Game Management, University of Natural Resources and Applied Life Sciences, Vienna, Gregor Mendel Str. 33, 1180 Vienna, Austria

5 Department for Ecology and Natural Resource Management, Norwegian University of Life Sciences, PO Box 5003, $1432 \AA ̊ ̊ s$, Norway

6 Norwegian Institute for Nature Research, 7485 Trondheim, Norway

7 Department of Wildlife, Fish and Environmental Studies, Swedish University of Agricultural Sciences, Umeå, Sweden 
because habitat selection links individuals to the resources required for survival and reproduction. Throughout their lives, individuals are constantly tasked to choose sets of resources (e.g. forage, prey, refuges) distributed within habitats to maximise their fitness (McLoughlin et al. 2010). When individual differences in habitat selection covary with fitness (McLoughlin et al. 2006; Leclerc et al. 2014), this variation, if heritable, represents alternative tactics available to adaptive evolution, which may change in frequency within a population according to density- or frequency-dependent selective pressures (Fortin et al. 2008). So far, however, no approach is available to explore the potential for evolution to act on individual differences in habitat selection behaviour. The first step to tackle this question is to document whether consistent individual variation in habitat selection exists.

Individual differences in behaviour have been studied for several decades (Krebs 1970; Bell et al. 2009). Originally, behaviours were assumed to potentially be completely plastic (Sih et al. 2004). More recently, however, behaviours are viewed as correlated traits that can generate trade-offs (Sih et al. 2004). Behavioural ecologists typically refer to those consistent individual differences as personality traits (Réale et al. 2010; Wolf and Weissing 2012). The study of individual differences in behaviour is of growing interest, because several studies have shown that such differences can have important ecological and evolutionary implications (Réale et al. 2010; Sih et al. 2012; Wolf and Weissing 2012). For example, individual variation in behaviour plays an important role in population dynamics in western bluebirds (Sialia mexicana), where aggressiveness and dispersal varies among males (Duckworth 2006; Duckworth and Badyaev 2007). Aggressive males disperse farther and colonise new habitats, whereas less aggressive males disperse less and have higher reproductive success in older established populations (Duckworth 2008). Therefore, for a given population, aggressiveness declines through time as the population becomes older (Duckworth 2008). Consistent individual differences in behaviour also have evolutionary implications, as selective pressures can act upon those differences, because they affect survival and reproduction (see review Smith and Blumstein 2008). For example, in North American red squirrels (Tamiasciurus hudsonicus), differences in female aggressiveness were correlated to overwinter offspring survival (Boon et al. 2007). The direction and strength of the relationship between behavioural traits and fitness can also depend on the environment (Nussey et al. 2007; Boon et al. 2007), highlighting the importance of studying consistent individual variation in habitat selection, which has yet to be done.

Morris (2003) defines habitat selection as the process whereby individuals use, or occupy, a nonrandom set of available habitats. Habitat selection is a hierarchical process (Johnson 1980), through which an individual aims to reduce the influence of limiting factors (a factor limiting an individual's fitness) (Rettie and Messier 2000; Leclerc et al. 2012). Consequently, habitat selection patterns may vary according to the spatial scale studied (Morris 1987; Meyer and Thuiller 2006). For example, at large spatial scales, yellow-headed blackbirds (Xanthocephalus xanthocephalus) place nests where food abundance is higher, but at a finer spatial scale they place nests where vegetation cover is greater (Orians and Wittenberger 1991). Therefore, careful attention to scale and limiting factors governing habitat selection are essential to accurately estimate biologically relevant behavioural patterns.

Patterns in habitat selection can also result from functional responses to habitat availability. Functional responses in habitat selection are defined as a change in the selection of a habitat type depending on its availability (Mysterud and Ims 1998). The study of functional responses can help our understanding of resource use trade-offs (Mabille et al. 2012), which in turn can influence fitness (Leclerc et al. 2014; Losier et al. 2015). Functional responses in habitat selection are often interpreted at the population level by looking at the habitat selection of individuals in different landscapes (e.g. Mabille et al. 2012). This usually occurs because one individual rarely exists in a variety of landscapes or in all landscapes available to the population during the study period (Fig. 1). Therefore, functional responses in habitat selection can be seen as a concept analogous to behavioural reaction norm (Fig. 1) and should be accounted for when evaluating consistent individual differences in habitat selection (Supplementary Material Fig. S1).

This study has three main objectives. First, we extend a method that combines ubiquitous practices from behavioural ecology, namely repeatability analysis and resource selection functions (RSFs), to quantify consistent individual differences in habitat selection. Second, we apply this method to a detailed behavioural dataset of GPS-collared brown bears (Ursus arctos) and assess whether individual differences in habitat selection are detectable. We focused our analyses on two habitat types, bogs and recent timber-harvest cut blocks (hereafter, cut blocks). We used bogs and cut blocks because they are the most abundant anthropogenically undisturbed and disturbed habitat types, respectively, in the study area and because they are avoided and selected for, respectively, at the population scale (Moe et al. 2007; Martin et al. 2010). Finally, using simulations, we explored the relationship between the repeatability in habitat selection across years and the strength at which a habitat type is selected or avoided at the population level. Ultimately, we argue that individual differences in habitat selection should be common in nature, given the evolutionary implications of resource choice strategies (see Fortin et al. 2008 for an example). 


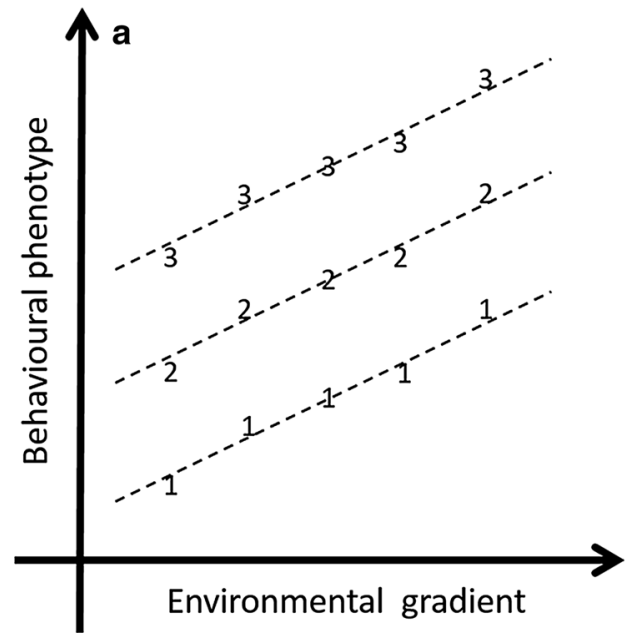

Fig. 1 Similarities can be drawn between the behavioural reaction norm (a) and the functional response in habitat selection (b) concepts. Both evaluate how the behaviour of individuals changes along an environmental gradient. Reaction norms are often evaluated with smaller species in laboratories or in open-field or maze tests. However, functional responses in habitat selection are usually interpreted at the population level, as individuals rarely exist in all landscapes

\section{Materials and methods}

The study area was located in south-central Sweden $\left(61^{\circ} \mathrm{N}\right.$, $15^{\circ} \mathrm{E}$ ) and was composed of bogs, lakes, and intensively managed coniferous forest stands of variable ages. The dominant tree species were Norway spruce Picea abies, Scots pine Pinus sylvestris, and birch Betula spp. Elevations ranged between 150 and $1000 \mathrm{~m}$ asl. Gravel roads $\left(0.7 \mathrm{~km} / \mathrm{km}^{2}\right)$ were more abundant than paved roads $\left(0.14 \mathrm{~km} / \mathrm{km}^{2}\right)$. See Martin et al. (2010) for further information about the study area.

We captured brown bears from a helicopter (2007-2012) using a remote drug delivery system (Dan-Inject, Børkop, Denmark). We extracted a vestigial first premolar for age determination from each individual not captured as a yearling with its mother (Matson 1993). We equipped bears with GPS collars (GPS Plus; Vectronic Aerospace, Berlin, Germany) programmed to relocate a bear every $30 \mathrm{~min}$. See Fahlman et al. (2011) for details on capture and handling. All bears captured were part of the Scandinavian Brown Bear Research Project, and all captures and handling were approved by the appropriate authority and ethical committee (Djuretiska nämden i Uppsala, Sweden).

\section{Spatial analysis}

The GPS location fix success rate was $>94 \%$. We screened the relocation data and removed GPS fixes with dilution of precision values $>5$ to increase spatial accuracy. Removed GPS locations were not biased with respect to habitat type

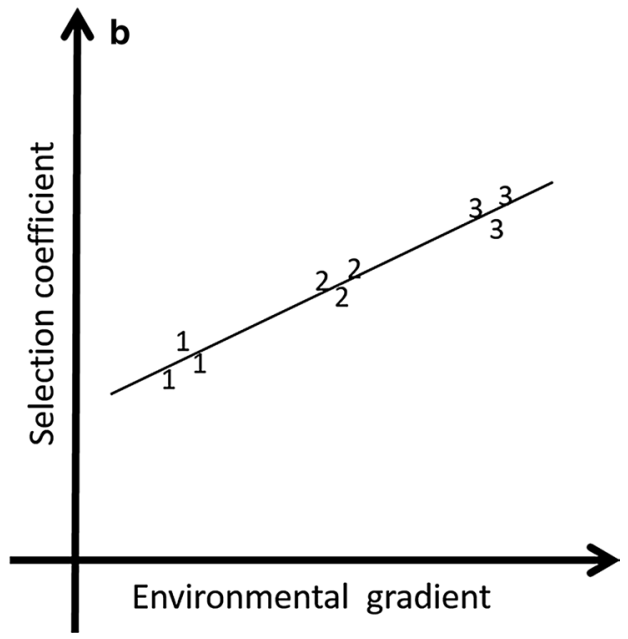

available to the population. Biased estimates of repeatability can be obtained if functional responses in habitat selection are not accounted for (Supplementary material, Fig. S1). Here, we assumed that if one individual would select habitat type " $X$ " (environmental gradient) less strongly than the mean population response, it would do so along the entire environmental gradient. Note that different numbers refer to different individuals

$(P>0.22)$ compared to GPS locations retained in our analyses. Preliminary analyses showed consistent results when working with $30 \mathrm{~min}, 1,2$, or $4 \mathrm{~h}$ locations intervals (data not shown). Therefore, we used the complete dataset, i.e. 30 min location intervals. We used GPS locations from 21 August to 20 September for males and lone females. We chose to use this time period, during which the bears are foraging on berries, to reduce the influence of seasonality on behaviour. Hereafter, the set of locations of one bear from 21 August to 20 September on a given year will be referred as bear-year.

For every bear-year, we selected the same number of random locations as GPS locations. Random locations were distributed within each bear-year's annual home range (3rd order of selection; sensu Johnson 1980). We defined home ranges as $100 \%$ minimum convex polygons (Mohr 1947). To consider the influence of the surrounding environment on habitat selection, we extracted covariates within a circular buffer with a 182-m radius (which corresponds to the mean distance between 2 GPS relocations) centred on each GPS and random location. Covariates were landscape characteristics known or expected to influence the probability of occurrence of bears, based on previous research (Moe et al. 2007; Martin et al. 2010; Steyaert et al. 2013) and were derived from Swedish Corine Land Cover $(25 \times 25 \mathrm{~m})$ and a Digital Elevation Model $(50 \times 50 \mathrm{~m})$ from National Land Survey of Sweden (licence i2012/901, www.lantmateriet.se). Covariates extracted from each buffer were the $\%$ coniferous stands (tree height $>5 \mathrm{~m}$ and canopy cover of conifers $>70 \%$ ), \% cut blocks (tree height 
$<2 \mathrm{~m}$ ), \% water, \% bogs (bogs with shrub and tree cover $<30 \%$ ), \% mixed-deciduous stands (tree height $>5 \mathrm{~m}$ and canopy cover of deciduous trees $>30 \%$ ), \% young forested stands (tree height 2-5 m), road length, mean elevation, and the coefficient of variation of elevation. We conducted all spatial analyses using ArcGIS 10.0 (ESRI, Redlands, CA, USA) and the Geospatial Modelling Environment 0.7.2 (Spatial Ecology).

\section{Statistical analysis}

We used RSFs (Manly et al. 2002) to assess habitat selection by conducting logistic regression that compared habitat characteristics at bear GPS locations (coded 1) to those at random locations (coded 0$)$. Habitat type covariates ( $\beta$ coefficients from the logistic regression) can be interpreted as selected or avoided if $\beta>0$ or $\beta<0$, respectively, and significantly different from 0 . If $\beta=0$, or is not significantly different from 0 , then the habitat type is used in proportion to its availability. More recently, RSFs often include individual as a random effect on the intercept and also include random coefficient (Gillies et al. 2006; Hebblewhite and Merrill 2008). Random intercepts account for differences in sample size among individuals, whereas random coefficients account for differences in selection among individuals (Gillies et al. 2006; Hebblewhite and Merrill 2008). To our knowledge, no study has used random coefficients in RSFs to test if habitat selection constitutes behaviour with consistent individual differences upon which natural selection could act. Prior to statistical analyses, we assessed multicolinearity between covariates using the variance inflation factor (VIF <5; Graham 2003), and, based on this, removed the \% coniferous stands from our analyses which occupied on average $>56 \%$ of buffer zones. We performed model selection (Burnham and Anderson 2002) and evaluated different candidate models defined a priori using the Akaike Information Criterion (AIC).

We first evaluated two different RSF models with differently structured random effects to ascertain whether variance in habitat selection occurred among individuals. In model A, we nested bear-year within BearID and included no random coefficient. This model provided information about habitat selection at the population level and accounted for differences in number of GPS fixes per individual (Gillies et al. 2006). In model B, we also considered differences in selection among individuals by adding $\%$ bog as a random coefficient to model A (Gillies et al. 2006). We added \% bog to test for individual variation in habitat selection toward an abundant natural habitat type, however, any other habitat covariate of interest could have been used instead (for cut blocks habitat type, see supplementary material). If supported by AIC, model $\mathrm{B}$ would permit us to extract variances in habitat selection of bogs (random coefficient) within (bear-year) and among (BearID) individuals to calculate repeatability:

$r=\frac{s_{\text {among }}^{2}}{s_{\text {among }}^{2}+s_{\text {within }}^{2}}$

where $r$ is repeatability, $s_{\text {among }}^{2}$ is the variance among individuals (BearID), and $s_{\text {within }}^{2}$ is the variance within individuals (bear-year). High repeatability $(r=1)$ will be found if $s_{\text {among }}^{2}$ is high relative to $s_{\text {within }}^{2}$, or, in other words, when individuals behave consistently through time (low $s_{\text {within }}^{2}$ ) and when individuals behave differently from each other (high $s_{\text {among }}^{2}$ ). No repeatability $(r=0)$ will be found when all individuals behave similarly as a homogenous group (low $s_{\text {among }}^{2}$ ), but the "group" behaves differently through time (high $s_{\text {within }}^{2}$ ).

Using the most parsimonious random structure, we evaluated 4 nested candidate models with different fixed effects. The 'base' model was composed of the functional response toward bogs only. Functional response was added by including an interaction term between the $\%$ bog within the $182-\mathrm{m}$ radius buffer and the $\%$ bog within the home range. The 'elevation' model included the 'base' model, mean elevation, and the coefficient of variation of elevation. The 'natural' model included the 'elevation' model, $\%$ water, and $\%$ mixed-deciduous stand. The 'full' model included the 'natural' model, \% cut blocks, \% young forested stand, and road length.

Subsequently, we estimated fixed and random coefficients from the most parsimonious model. We extracted variance of bog selection among bears (BearID) and within bears (bear-year) and calculated repeatability according to Eq. 1. To facilitate model convergence, all numeric covariates were scaled (mean $=0$, variance $=1$ ) before inclusion. We conducted all statistical analyses using the lme4 package (Bates et al. 2015) in R 3.0.1 (R Core Team 2013).

\section{Simulations}

We performed simulations to ensure that repeatability estimates calculated from the random effects of RSF were not functions of the value of $\beta$ estimates or their significance. Three scenarios were tested (Supplementary material, Appendix S1). In each scenario, we created a population of five individuals living in similar landscapes and monitored for 3 years. In the first scenario, parts of the population always selected habitat type $\mathrm{X}$, whereas others always avoided it with varying intensities among years. In the second scenario, all individuals in the population avoided, used in proportion to availability, and selected habitat type $\mathrm{X}$ in the first, second, and third year, respectively, but we did not allow variation among individuals in a given year. In the third scenario, all individuals in the population selected 
habitat type $\mathrm{X}$ with varying intensity between years, but we did not allow variation among bears in a given year. We evaluated repeatability estimates for each scenario (Supplementary material, Appendix S1) using the lme4 package (Bates et al. 2015) in R 3.0.1 (R Core Team 2013).

\section{Results}

Between 2007 and 2012, we followed 31 GPS collared bears, 12 males and 19 lone females, aged 2-20 years-old. The bears were tracked $\bar{x}=2.81$ years (range: $2-5$ years) for a total of 87 bear-years, which included a total of 72,744 GPS locations (mean $=836$ GPS locations per bear-year). Annual home range availability of bogs differed between bears and bear-year $(\bar{x}=13 \%$, range $=2-27 \%$ of annual home ranges).

We evaluated two random structures. Adding a random coefficient for \% bog in the RSF increased model support (Table 1), suggesting that differences existed in the selection of bogs between BearID and/or bear-year. For the selection of fixed effects, the 'full' model had the strongest support (Table 2). The fixed effect showed that, at the population level, bears selected for cut blocks, young forest, mixed-deciduous stands, and high coefficient of variation

Table 1 Random structures tested to assess the repeatability of habitat selection of bogs by brown bears (Ursus arctos) in Sweden between 2007 and 2012

\begin{tabular}{llllrr}
\hline Model & $\begin{array}{l}\text { Random } \\
\text { intercept }\end{array}$ & $\begin{array}{l}\text { Random } \\
\text { coefficient }\end{array}$ & LL & $\Delta$ AIC & $w_{\mathrm{i}}$ \\
\hline A & $\begin{array}{l}\text { Bear-year } \\
\text { nested in BearID }\end{array}$ & - & $-92,098$ & 1760 & 0 \\
B & $\begin{array}{c}\text { Bear-year } \\
\text { nested in BearID }\end{array}$ & $\%$ bog & $-91,214$ & 0 & 1 \\
\hline
\end{tabular}

Models are listed with their random intercepts, random coefficient, $\log$ likelihood $(L L)$, differences in Akaike Information Criterion in relation to the best-supported model $(\triangle A I C)$, and Akaike weight $\left(w_{\mathrm{i}}\right)$ of elevation, but avoided high road density, water, and bogs (Table 3).

We estimated the repeatability of bog selection by extracting within (bear-year) and among (BearID) bear variances from the 'full' model. Variance of bog selection within (bear-year) and among (BearID) bears was 0.081 and 0.035 , respectively. According to Eq. 1, repeatability of bog selection was 0.304 , indicating that $30.4 \%$ of the variance in habitat selection of bogs by bears was due to differences among individuals (Fig. 2). Habitat selection of cut blocks showed similar results (Supplementary material, Tables S1-S3). Variation in selection of cut blocks within (bear-year) and among (BearID) bears was 0.034 and 0.025 , respectively, resulting in a repeatability of 0.420 (Fig. 2).

The simulation results suggested that in scenario 1, habitat type $\mathrm{X}$ was neither selected nor avoided at the population level, but was highly repeatable at the individual level ( $>0.8$; Table 4; Supplementary material, Appendix S1). Habitat type $X$ from scenario 2 was also neither selected nor avoided at the population level, but was not repeatable $(<0.001$; Table 4; Supplementary material, Appendix S1). Finally, in scenario 3 , habitat type $\mathrm{X}$ was selected at the population level but was not repeatable $(<0.001$; Table 4; Supplementary material, Appendix S1).

\section{Discussion}

Although natural selection acts on individual phenotypes, most literature on habitat selection reports population-scale inferences. Here, we have extended a traditional method based on RSF to investigate habitat selection at the individual level and have shown that individual variation in habitat selection exists in our brown bear study population. By investigating habitat selection at the individual level, we found that individual differences in habitat selection existed, were repeatable, and revealed patterns in selection that were not apparent at the population level. Bears avoided bogs at the population level, but with varying

Table 2 Candidate models tested to assess repeatability of habitat selection of bogs by brown bears in Sweden between 2007 and 2012

\begin{tabular}{|c|c|c|c|c|}
\hline Model & Covariate included & LL & $\Delta \mathrm{AIC}$ & $w_{\mathrm{i}}$ \\
\hline Base & $\%$ bog $+\%$ bog in the annual home range $+\%$ bog $\times \%$ bog in the annual home range & $-96,521$ & 10,599 & 0 \\
\hline Elevation & Base model + mean elevation + coefficient of variation of elevation & $-95,946$ & 9454 & 0 \\
\hline Natural $^{\mathrm{a}}$ & Elevation model $+\%$ water $+\%$ mixed-deciduous & $-93,921$ & 5407 & 0 \\
\hline Full & Natural model $+\%$ cut blocks $+\%$ young forest + road density & $-91,214$ & 0 & 1 \\
\hline
\end{tabular}

Models are listed with their fixed effects covariates, log likelihood $(L L)$, differences in Akaike Information Criterion in relation to the bestsupported model $(\triangle A I C)$, and Akaike weight $\left(w_{\mathrm{i}}\right)$. All models were tested with bear-year nested in BearID as a random intercept and $\%$ bog as a random coefficient (model B from Table 1)

a The $\%$ of coniferous stands was not included to avoid multicolinearity (see "Materials and methods") 
Table 3 Coefficients $(\beta)$ and $95 \%$ confidence intervals of the fixed effect covariates of the most parsimonious model to assess the repeatability of bog habitat selection by brown bears in Sweden between 2007 and 2012

\begin{tabular}{|c|c|c|c|}
\hline \multirow[t]{2}{*}{ Variable } & \multirow[t]{2}{*}{$\beta$} & \multicolumn{2}{|c|}{$\begin{array}{l}95 \% \text { confidence } \\
\text { interval }\end{array}$} \\
\hline & & Lower & Upper \\
\hline Intercept & -0.078 & -0.132 & -0.024 \\
\hline$\%$ Bog & -0.514 & -0.606 & -0.422 \\
\hline$\%$ Bog within annual home range & 0.089 & 0.045 & 0.133 \\
\hline Mean elevation & -0.110 & -0.128 & -0.092 \\
\hline Coefficient of variation of elevation & 0.080 & 0.068 & 0.093 \\
\hline$\%$ Water & -0.477 & -0.496 & -0.458 \\
\hline$\%$ Mixed-deciduous & 0.018 & 0.007 & 0.029 \\
\hline$\%$ Young forest & 0.168 & 0.156 & 0.180 \\
\hline$\%$ Cut blocks & 0.272 & 0.260 & 0.284 \\
\hline Roads length & -0.306 & -0.317 & -0.294 \\
\hline $\begin{array}{l}\% \text { Bogs } \times \% \text { bogs within annual home } \\
\text { range }\end{array}$ & -0.074 & -0.158 & 0.010 \\
\hline
\end{tabular}

This model also included bear-year nested in BearID as a random intercept and $\%$ bog as a random coefficient (model B from Table 1)

intensity. Some bears avoided bogs more strongly than others. A similar pattern was also observed for cut blocks. Cut blocks were selected for at the population level, but consistent individual differences in their selection occurred among bears. Our simulations also suggested that repeatability estimates were not influenced by the pattern of habitat selection at the population level.

In our study, we have focused on individual variation in habitat selection toward habitat types that were selected and avoided at the population level. However, we expect that individual variation in habitat selection can also occur regarding habitat types that appear to be used in proportion to their availability at the population level, i.e. in habitat types with a non-significant $\beta$ estimate in RSFs. For example, we should observe individual variation in habitat selection toward a 'non-significant' habitat type if individuals behave differently from one to another, but the mean population use is equivalent to the mean population availability (Table 4; see simulations in Supplementary material, Appendix S1). Furthermore, if a habitat type is selected or avoided at the population level, i.e. $\beta$ estimate $\neq 0$, this does not imply that selection for or avoidance of this habitat type will be repeatable at the individual level. For example, all individuals in a population could express the same behaviour (low among-individual variation relative to withinindividual variation) of avoiding or selecting a habitat type, resulting in a low or zero repeatability (Table 4; see simulations in Supplementary material, Appendix S1). We therefore do not expect a relationship between the value and significance of $\beta$ estimates in RSFs and their repeatability.
The biological significance of individual differences in habitat selection will be linked to the spatial scale at which a study is conducted. Here, we evaluated habitat selection at the third order of selection (Johnson 1980), where bears should be less influenced by conspecifics and selection should reflect their own trade-offs regarding resource use (see Steyaert et al. 2013 for the mating period). If we had evaluated habitat selection repeatability at the second order of selection, we might have evaluated the consistency of the social structure and intra-specific competition rather than resource use trade-offs (Dahle and Swenson 2003; Støen et al. 2005; Dahle et al. 2006). In addition to choosing the most biologically relevant spatial scale, careful attention must be paid to density-dependent habitat selection (van Beest et al. 2014). Based on Ideal Free Distribution theory, individuals should distribute themselves to reduce resource competition and maximise fitness (Fretwell and Lucas 1970). Favourable habitat types should be used less by individuals when density increases, leading to a generalisation in habitat selection (Fortin et al. 2008; van Beest et al. 2014). Therefore, observed habitat selection patterns and repeatability estimates can be functions of varying density over time (lower repeatability) or across the landscape (higher repeatability). We did not control for bear density, as we assumed that it was stable over the study area during the study period (6 years). Furthermore, bears typically show a despotic distribution (Elfström et al. 2014), and density should influence habitat selection of bears at the second, rather than the third, order of selection. Briefly, careful attention must be paid to density-dependent habitat selection and the spatial scale at which we evaluated habitat selection repeatability, which should vary depending on a species' ecology, limiting factors, etc.

Consistent individual variation in behaviour, or animal personality, has been shown to occur across many species for a variety of behaviours (Bell et al. 2009). In a metaanalysis, the average repeatability across all behaviours was 0.37 (Bell et al. 2009), which is similar to the habitat selection repeatability estimates that we obtained. Traditional experiments of personality have consisted mainly of capturing individuals in the wild and quantifying their behaviours in laboratory or open field tests (Bell et al. 2009). Niemelä and Dingemanse (2014) argued that novel environments (e.g. in a laboratory) can elicit behavioural patterns that fail to match behaviours expressed in natural environments. By using remotely sensed data (i.e. GPS collars), we avoid this criticism, having measured behaviour directly in the wild. The advent of technologies, such as GPS telemetry (or camera traps, Passive Integrated Transponder networks, etc.), presents a plethora of opportunities for understanding the repeatability of a diverse range of animal behaviours, e.g. here with habitat selection (see also Ciuti et al. 2012; Kays et al. 2015; Wilmers et al. 2015). 

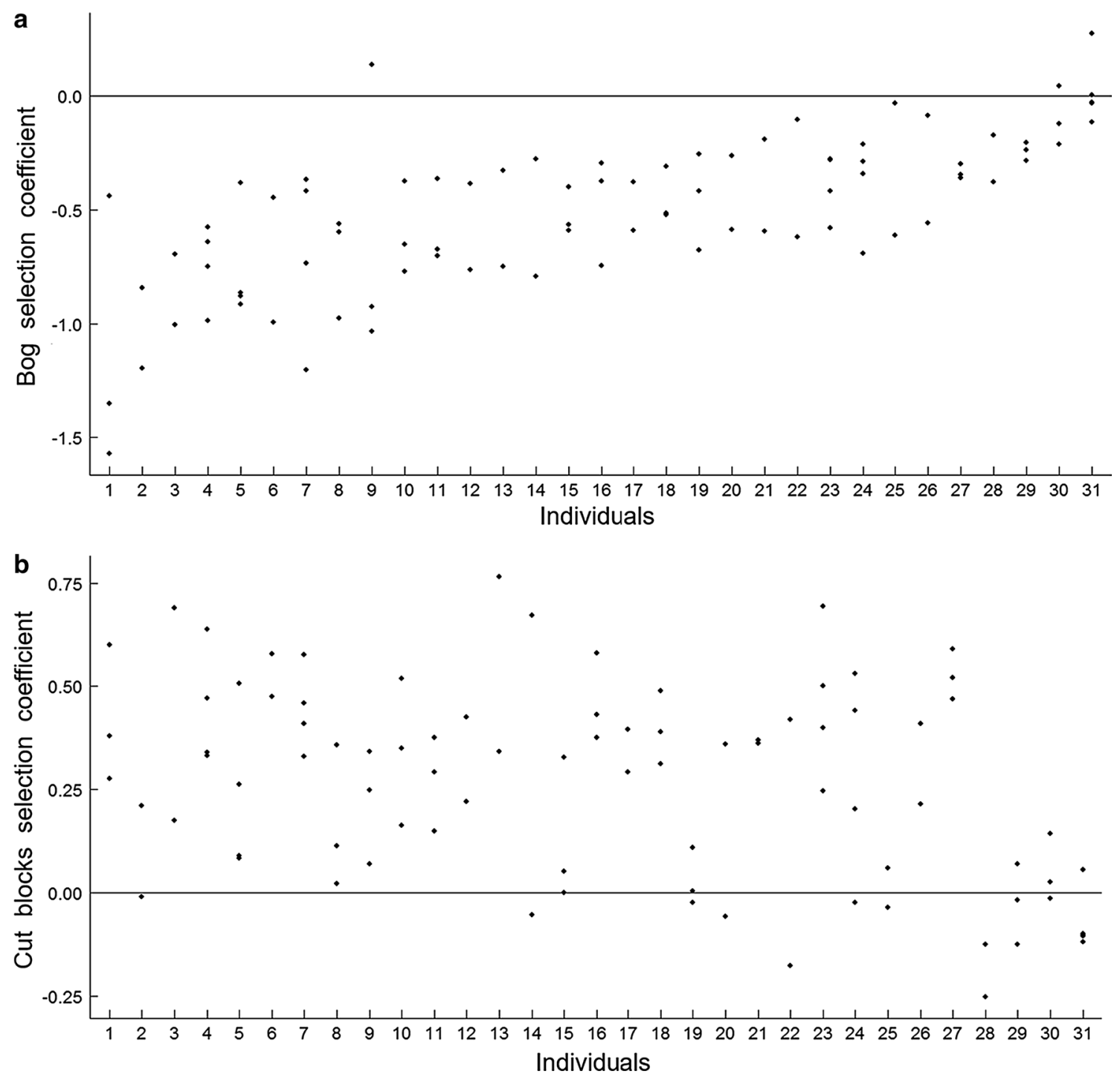

Fig. 2 Estimates of coefficients for the selection of bogs (a) and cut blocks (b) for each bear-year $(n=87)$ of brown bears (Ursus arctos) in south-central Sweden from 2007 to 2012. Bear-year is represented by a single dot, whereas stacked dots represent selection coefficients of a given individual $(n=31)$. Some bears consistently avoided bogs

Coupling measurements coming from both traditional experiments and personality measures using GPS telemetry will provide new opportunities to assess whether behaviour measured in laboratory or open field tests is associated with behaviour in the wild.

Consistent individual differences in habitat selection may have important ecological and evolutionary implications. As the expression of personality traits can be environment-dependent (Nussey et al. 2007), we suggest that individual variation in habitat selection could have important cascading effects on other behavioural traits (Dubois and Giraldeau 2014). For example, individual variation in habitat selection might canalise individuals into different or selected cut blocks more strongly than others. The repeatability estimate of bog and cut blocks selection coefficients was 0.304 and 0.420 , respectively, which indicates that individual variation in habitat selection exists and may allow adaptive evolution to occur in this brown bear population

behavioural patterns. In return, those behavioural patterns might appear as personality traits that could be caused by individual variation in habitat selection. More research linking habitat selection and animal personality is needed to disentangle the causes and consequences of individual variation in habitat selection and its potential cascading effects on other behavioural traits.

Individual differences in habitat selection could be the results of many non-exclusive factors. As bears seek resources that are distributed into habitat types, differences in habitat selection pattern could be the result of different resource needs in relation to sex or age. Therefore, it might not be surprising that we observed high inter-annual 
Table 4 Summary of results from simulations and GPS-collared bears that explored the relationship between the repeatability of habitat selection and the value and significance of $\beta$ estimates in RSFs

$$
\begin{aligned}
& \begin{array}{l}
\text { Population level RSFs } \beta \text { estimate Repeatability } \\
\text { response }
\end{array} \\
& \text { R }
\end{aligned}
$$

\begin{tabular}{cccc}
\hline $\begin{array}{c}\text { Simulations } \\
\text { Scenario } 1\end{array}$ & $\begin{array}{c}\text { Not selected nor } \\
\text { avoided }\end{array}$ & Non-significant & $>0.8$ \\
Scenario 2 & $\begin{array}{c}\text { Not selected nor } \\
\text { avoided }\end{array}$ & Non-significant & $<0.001$ \\
Scenario 3 & Selected & Significant & $<0.001$ \\
GPS-collared bear data & Significant & 0.304 \\
$\%$ Bog & Avoided & Significant & 0.420 \\
\% Cut blocks Selected & &
\end{tabular}

In each scenario, 5 individuals were followed for 3 years and existed in similar landscapes and expressed different habitat selection pattern. For further details on scenarios, see supplementary material Appendix S1

variance (bear-year) in habitat selection, as bears are opportunistic omnivores and the distribution of resource availability can differ among years (Bojarska and Selva 2012). Another mechanism that could explain individual differences in habitat selection is natal habitat preference induction, i.e. when experience in a natal habitat increases the level of preference for that habitat later in life (Davis and Stamps 2004; Stamps et al. 2009). Similarly, Nielsen et al. (2013) suggested that habitat selection in brown bears is a behaviour learned from the mother. Finally, as repeatability estimates are considered to be the upper limit of heritability (Falconer and Mackay 1996), our results suggest that patterns of habitat selection may be, at least partly, heritable (Shafer et al. 2014). Thus, we speculate that if these individual differences in habitat selection have a genetic basis and are under selective pressure, we would expect evolutionary change in patterns of habitat selection, which may have important implications for adaptive potential and the maintenance of genetic variation in wild populations.

Acknowledgments We thank Pierre-Olivier Montiglio for statistical advices and one anonymous reviewer for constructive comments. ML was supported financially by NSERC, FQRNT, and NSTP. EVW was supported financially by an NSERC postdoctoral fellowship. FP was funded by NSERC discovery grant and by the Canada research Chair in Evolutionary Demography and Conservation. This is scientific publication No. 194 from the SBBRP, which was funded by the Swedish Environmental Protection Agency, the Norwegian Directorate for Nature Management, the Research Council of Norway, and the Austrian Science Fund. We acknowledge the support of the Center for Advanced Study in Oslo, Norway, that funded and hosted our research project "Climate effects on harvested large mammal populations" during the academic year of 2015-2016 and funding from the Polish-Norwegian Research Program operated by the National Center for Research and Development under the Norwegian Financial Mechanism 2009-2014 in the frame of Project Contract No POL-NOR/198352/85/2013.
Author contribution statement ML and EVW developed the idea. All authors participated in the study design. ML carried out analyses. All authors wrote the manuscript and gave final approval for publication. AZ and JK participated in the coordination of the Scandinavian Brown Bear Research Project (SBBRP). JES coordinated the SBBRP.

Open Access This article is distributed under the terms of the Creative Commons Attribution 4.0 International License (http://creativecommons.org/licenses/by/4.0/), which permits unrestricted use, distribution, and reproduction in any medium, provided you give appropriate credit to the original author(s) and the source, provide a link to the Creative Commons license, and indicate if changes were made.

\section{References}

Bates D, Maechler M, Bolker B, Walker S (2015) Fitting linear mixed-effects models using lme4. J Stat Softw 67:1-48. doi:10.18637/jss.v067.i01

Bell AM, Hankison SJ, Laskowski KL (2009) The repeatability of behaviour: a meta-analysis. Anim Behav 77:771-783. doi:10.1016/j.anbehav.2008.12.022

Bojarska K, Selva N (2012) Spatial patterns in brown bear Ursus arctos diet: the role of geographical and environmental factors. Mamm Rev 42:120-143. doi:10.1111/j.1365-2907.2011.00192.x

Boon AK, Réale D, Boutin S (2007) The interaction between personality, offspring fitness and food abundance in North American red squirrels. Ecol Lett 10:1094-1104. doi:10.1111/j.1461-0248.2007.01106.x

Burnham KP, Anderson DR (2002) Model selection and multimodel inference, 2nd edn. Springer, Berlin

Ciuti S, Muhly TB, Paton DG et al (2012) Human selection of elk behavioural traits in a landscape of fear. Proc R Soc Lond B 279:4407-4416. doi:10.1098/rspb.2012.1483

Dahle B, Swenson JE (2003) Home ranges in adult Scandinavian brown bears (Ursus arctos): effect of mass, sex, reproductive category, population density and habitat type. J Zool 260:329-335. doi:10.1017/S0952836903003753

Dahle B, Støen O-G, Swenson JE (2006) Factors influencing homerange size in subadult brown bears. J Mammal 87:859-865. doi:10.1644/05-MAMM-A-352R1.1

Davis JM, Stamps JA (2004) The effect of natal experience on habitat preferences. Trends Ecol Evol 19:411-416. doi:10.1016/j. tree.2004.04.006

Dubois F, Giraldeau L-A (2014) How the cascading effects of a single behavioral trait can generate personality. Ecol Evol 4:30383045. doi:10.1002/ece3.1157

Duckworth RA (2006) Behavioral correlations across breeding contexts provide a mechanism for a cost of aggression. Behav Ecol 17:1011-1019. doi:10.1093/beheco/arl035

Duckworth RA (2008) Adaptive dispersal strategies and the dynamics of a range expansion. Am Nat 172:S4-S17. doi:10.1086/588289

Duckworth RA, Badyaev AV (2007) Coupling of dispersal and aggression facilitates the rapid range expansion of a passerine bird. Proc Natl Acad Sci USA 104:15017-15022. doi:10.1073/ pnas.0706174104

Elfström M, Zedrosser A, Støen O-G, Swenson JE (2014) Ultimate and proximate mechanisms underlying the occurrence of bears close to human settlements: review and management implications. Mamm Rev 44:5-18. doi:10.1111/j.1365-2907.2012.00223.x

Fahlman A, Arnemo JM, Swenson JE et al (2011) Physiologic Evaluation of Capture and Anesthesia with Medetomidine-Zolazepam-Tiletamine in Brown Bears (Ursus arctos). J Zoo Wildl Med 42:1-11. doi:10.1638/2008-0117.1 
Falconer DS, Mackay TFC (1996) Introduction to quantitative genetics, 4th edn. Benjamin Cummings, San Francisco

Fortin D, Morris DW, McLoughlin PD (2008) Habitat selection and the evolution of specialists in heterogeneous environments. Isr $\mathbf{J}$ Ecol Evol 54:311-328. doi:10.1560/IJEE.54.3-4.311

Fretwell SD, Lucas HJ (1970) On territorial behavior and other factors influencing habitat distributions in birds. Acta Biotheor 19:16-36. doi:10.1007/BF01601953

Gillies CS, Hebblewhite M, Nielsen SE et al (2006) Application of random effects to the study of resource selection by animals. J Anim Ecol 75:887-898. doi:10.1111/j.1365-2656.2006.01106.x

Graham MH (2003) Confronting multicollinearity in ecological multiple regression. Ecology 84:2809-2815. doi:10.1890/02-3114

Hebblewhite M, Merrill E (2008) Modelling wildlife-human relationships for social species with mixed-effects resource selection models. J Appl Ecol 45:834-844. doi:10.1111/j.1365-2664.2008.01466.x

Johnson DH (1980) The comparison of usage and availability measurements for evaluating resource preference. Ecology 61:65-71. doi: $10.2307 / 1937156$

Kays R, Crofoot MC, Jetz W, Wikelski M (2015) Terrestrial animal tracking as an eye on life and planet. Science 348:2478. doi:10.1126/science.aaa2478

Krebs CJ (1970) Microtus population biology: behavioral changes associated with the population cycle in $\mathrm{M}$. ochrogaster and $\mathrm{M}$. pennsylvanicus. Ecology 51:34-52. doi:10.2307/1933598

Leclerc M, Dussault C, St-Laurent M-H (2012) Multiscale assessment of the impacts of roads and cutovers on calving site selection in woodland caribou. For Ecol Manag 286:59-65. doi:10.1016/j. foreco.2012.09.010

Leclerc M, Dussault C, St-Laurent M-H (2014) Behavioural strategies towards human disturbances explain individual performance in woodland caribou. Oecologia 176:297-306. doi:10.1007/ s00442-014-3012-9

Losier CL, Couturier S, St-Laurent M-H et al (2015) Adjustments in habitat selection to changing availability induce fitness costs for a threatened ungulate. J Appl Ecol 52:496-504. doi:10.1111/1365-2664.12400

Mabille G, Dussault C, Ouellet J-P, Laurian C (2012) Linking tradeoffs in habitat selection with the occurrence of functional responses for moose living in two nearby study areas. Oecologia 170:965-977. doi:10.1007/s00442-012-2382-0

Manly BFJ, McDonald LL, Thomas DL et al (2002) Resource selection by animals: Statistical analysis and design for field studies, 2nd edn. Kluwer, Boston

Martin J, Basille M, Van Moorter B et al (2010) Coping with human disturbance: spatial and temporal tactics of the brown bear (Ursus arctos). Can J Zool 88:875-883. doi:10.1139/Z10-053

Matson GM (1993) A laboratory manual for cementum age determination of Alaska brown bear first premolar teeth. Alaska Department of Fish and Game and Matson's Laboratory, Milltown, Montana

McLoughlin PD, Boyce MS, Coulson T, Clutton-Brock T (2006) Lifetime reproductive success and density-dependent, multivariable resource selection. Proc R Soc Lond B 273:1449-1454. doi:10.1098/rspb.2006.3486

McLoughlin PD, Morris DW, Fortin D et al (2010) Considering ecological dynamics in resource selection functions. J Anim Ecol 79:4-12. doi:10.1111/j.1365-2656.2009.01613.x

Meyer CB, Thuiller W (2006) Accuracy of resource selection functions across spatial scales. Divers Distrib 12:288-297. doi:10.1111/j.1366-9516.2006.00241.x

Moe TF, Kindberg J, Jansson I, Swenson JE (2007) Importance of diel behaviour when studying habitat selection: examples from female Scandinavian brown bears (Ursus arctos). Can J Zool 85:518-525. doi:10.1139/Z07-034
Mohr CO (1947) Table of equivalent populations of North American small mammals. Am Midl Nat 37:223-249. doi:10.2307/2421652

Morris DW (1987) Ecological scale and habitat use. Ecology 68:362369. doi: $10.2307 / 1939267$

Morris DW (2003) Toward an ecological synthesis: a case for habitat selection. Oecologia 136:1-13. doi:10.1007/s00442-003-1241-4

Morris DW (2011) Adaptation and habitat selection in the ecoevolutionary process. Proc R Soc Lond B 278:2401-2411. doi:10.1098/rspb.2011.0604

Mysterud A, Ims RA (1998) Functional responses in habitat use: availability influences relative use in trade-off situations. Ecology 79:1435-1441. doi:10.1890/0012-9658(1998)079[1435:FRI HUA]2.0.CO;2

Nielsen SE, Shafer ABA, Boyce MS, Stenhouse GB (2013) Does learning or instinct shape habitat selection? PLoS ONE 8:e53721. doi:10.1371/journal.pone.0053721

Niemelä PT, Dingemanse NJ (2014) Artificial environments and the study of "adaptive" personalities. Trends Ecol Evol 29:245-247. doi:10.1016/j.tree.2014.02.007

Nussey DH, Wilson AJ, Brommer JE (2007) The evolutionary ecology of individual phenotypic plasticity in wild populations. J Evol Biol 20:831-844. doi:10.1111/j.1420-9101.2007.01300.x

Orians GH, Wittenberger JF (1991) Spatial and temporal scales in habitat selection. Am Nat 137:S29-S49. doi:10.1086/285138

R Core Team (2013) R: a language and environment for statistical computing. R Foundation for Statistical Computing, Vienna. http://www.R-project.org/

Réale D, Dingemanse NJ, Kazem AJN, Wright J (2010) Evolutionary and ecological approaches to the study of personality. Philos Trans R Soc Lond B 365:3937-3946. doi:10.1098/ rstb.2010.0222

Rettie WJ, Messier F (2000) Hierarchical habitat selection by woodland caribou: its relationship to limiting factors. Ecography 23:466-478. doi:10.1034/j.1600-0587.2000.230409.x

Shafer ABA, Nielsen SE, Northrup JM, Stenhouse GB (2014) Linking genotype, ecotype, and phenotype in an intensively managed large carnivore. Evol Appl 7:301-312. doi:10.1111/eva.12122

Sih A, Bell AM, Ziemba RE (2004) Behavioral syndromes: an integrative overview. Q Rev Biol 79:241-277. doi:10.1086/422893

Sih A, Cote J, Evans M et al (2012) Ecological implications of behavioural syndromes. Ecol Lett 15:278-289. doi:10.1111/j.1461-0248.2011.01731.x

Smith BR, Blumstein DT (2008) Fitness consequences of personality: a meta-analysis. Behav Ecol 19:448-455. doi:10.1093/beheco/ $\operatorname{arm} 144$

Stamps JA, Krishnan VV, Willits NH (2009) How different types of natal experience affect habitat preference. Am Nat 174:623-630. doi: $10.1086 / 644526$

Steyaert SMJG, Kindberg J, Swenson JE, Zedrosser A (2013) Male reproductive strategy explains spatiotemporal segregation in brown bears. J Anim Ecol 82:836-845. doi:10.1111/1365-2656.12055

Støen O-G, Bellemain E, Sæbø S, Swenson JE (2005) Kin-related spatial structure in brown bears Ursus arctos. Behav Ecol Sociobiol 59:191-197. doi:10.1007/s00265-005-0024-9

Van Beest FM, Uzal A, Vander Wal E et al (2014) Increasing density leads to generalization in both coarse-grained habitat selection and fine-grained resource selection in a large mammal. J Anim Ecol 83:147-156. doi:10.1111/1365-2656.12115

Wilmers CC, Nickel B, Bryce CM et al (2015) The golden age of biologging: how animal-borne sensors are advancing the frontiers of ecology. Ecology 96:1741-1753. doi:10.1890/14-1401.1

Wolf M, Weissing FJ (2012) Animal personalities: consequences for ecology and evolution. Trends Ecol Evol 27:452-461. doi:10.1016/j.tree.2012.05.001 